\title{
Ant Colony Optimization for Resolving Unit Commitment Issues by Considering Reliability Constraints
}

\author{
Alan Abdu Robbi Afifi ${ }^{1}$, Sarjiya ${ }^{2}$, Yusuf Susilo Wijoyo ${ }^{3}$
}

\begin{abstract}
Unit Commitment or generator scheduling is one of complex combination issues aiming to obtain the cheapest generating power total costs. Ant Colony Optimization is proposed as a method to solve Unit Commitment issues because it has a better result convergence according to one of journals that reviews methods to solve Unit Commitment issues. Ant Colony Optimization modification into Nodal Ant Colony Optimization as well as addition of several elements are also conducted to overcome Ant Colony Optimization limitations in resolving Unit Commitment issues. Nodal Ant Colony Optimization simulations are then compared with Genetic Algorithm and Simulated Annealing methods which previously has similar simulations. Reliability index combination in a form of Loss of Load Probability and Expected Unserved Energy are also added as reliability constraints in the system. Comparison of three methods shows that Nodal Ant Colony Optimization is able to provide better results up to $0.08 \%$ cheaper than Genetic Algorithm or Simulated Annealing methods.
\end{abstract}

Keywords - Generator Scheduling, Nodal Ant Colony Optimization, Reliability Constraints.

\section{INTRODUCTION}

The Unit Commitment (UC) is a decision-making process used to determine optimal generator scheduling by minimizing required total operating costs. Importance of UC problems results is that it is used as a resolution of load demand cycle occurring on daily basis. UC issues in a large scale have relatively complex problems in finding their resolution. Several studies that reviewed UC resolution methods explained the effectiveness and advantages of each method in solving UC problems, one of which stated that Ant Colony Optimization (ACO) and PSO methods were highlighted because the results given were more convergent [1].

ACO is an optimization method that mimics ant behavior in determining the shortest distance between nests to food source. Some studies show that ACO has a better solution than other methods used in the study [2]-[4]. Nevertheless, the use of ACO as a UC solution has not been used in scheduling a largescale power generator because it still uses ten generating units as a test system.

One of causes of UC issues complexity is that generator number addition in system will cause total combination of

${ }^{1}$ Department of Electrical Engineering and Information Technology, Gadjah Mada University, Jl. Grafika No. 2 UGM Campus, Yogyakarta, 55281 INDONESIA (email: abdualan@gmail.com)

2,3 Lecturer, Department of Electrical Engineering and Information Technology, Gadjah Mada University, Jl. Grafika No. 2 UGM Campus, Yogyakarta, 55281 INDONESIA (email: sarjiya@ugm.ac.id,yusufsw@ugm.ac.id) generator scheduling increase exponentially. For example, if the possibility of a generator is only an on/off status (1 and 0 ), then the possibility of total generator that can be obtained for each hour is (2Nרา-1), with $N$ as generator unit number. Those number of combinations which later becomes a problem in ACO method, because ACO use is very dependent on ant line formations originating from combination of those generators.

This research implements a Nodal Ant Colony Optimization (NACO) method in its use. The utilized NACO concept in this study remains the same as NACO which comes from [5]. The difference is that NACO utilized in this paper adopts the best cost per produced unit function instead of fuel cost visibility value [6].

Simulations carried out in this research also add constraints reliability into it. System reliability level is then represented in reliability index in a form of Loss of Load Probability (LOLP) and Expected Unserved Energy (EUE). LOLP and EUE are chosen because those two indices show probability magnitude and amount of energy that is not met at a certain time period. In addition, utilization of both indices was also used in previous studies, which ease the comparison process between Genetic Algorithm (GA) [7] and a Simulated Annealing (SA) [8] methods simulation results [9].

\section{PROBlem Formulation}

\section{A. Unit Commitment}

The objective function of UC is to minimize required total generation costs. UC objective formulation from UC only takes two elements, namely fuel cost and start-up cost functions. These two functions are then optimized so that minimum generation cost is obtained.

$$
T C=\sum_{t=1}^{T} \sum_{i=1}^{N} U_{i, t} F C_{i}\left(P_{i, t}\right)+U_{i, t} S U_{i, t}
$$

with

$$
\begin{aligned}
& F C_{i}\left(P_{i, t}\right)=a_{i} P_{i, t}^{2}+b_{i} P_{i, t}+c_{i} \\
& S U_{i, t}=C_{c}\left(1-e^{-t / \alpha}\right)+C_{f}
\end{aligned}
$$

with

$$
\begin{aligned}
& \text { i } \quad \text { = generating unit; } \\
& t \quad=\text { time (hour); } \\
& T \quad=\text { total UC scheduling time (hour); } \\
& N=\text { = total number of generating units; } \\
& \text { TC = total cost of generating operation } \\
& U_{i, t} \quad=\text { condition of generating unit number }-i \text { at } t
\end{aligned}
$$




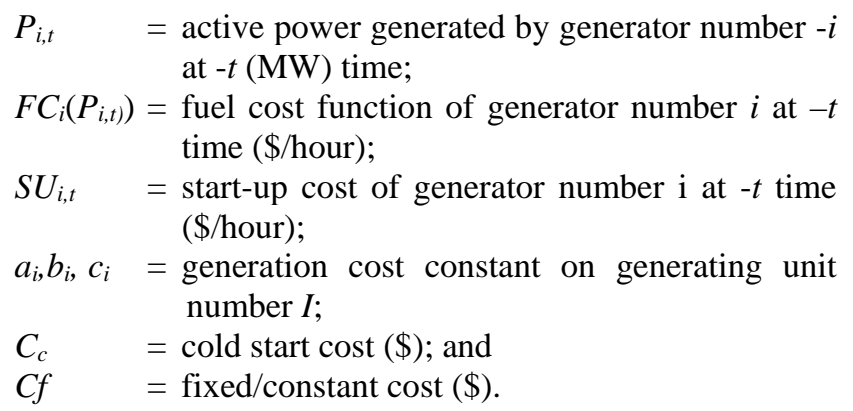

Some limitations which need to be considered in completing generating unit schedule are as follows.

- Active power balance:

$$
\sum_{i \in N} U_{i, t} P_{i, t}=P_{D, t} \quad t \in T .
$$

- Maximum and minimum power per generator

$$
U_{i, t} P_{\min , i} \leq P_{i, t} \leq U_{i, t} P_{\text {maks }, i} .
$$

- Minimum uptime and downtime of generating units

$$
\begin{gathered}
X_{i}^{\text {on }}(t) \geq M U T_{i}, \\
X_{i}^{\text {off }}(t) \geq M D T_{i, \cdot}
\end{gathered}
$$

\section{B. System Reliability}

Addition of system Reliability level aims to evaluate scheduling results toward the tested reliability level. A system is claimed to be reliable if failure possibility of the generator and system can be anticipated so that all loads remain fulfilled in a certain period of time. Some variables used to formulate the system reliability levels are as follows.

1) Outage Replacement Rate (ORR): ORR indicates probability of a unit to fail and that unit cannot be substituted during lead time $(T)$.

$$
\text { Unavaibility }=1-e^{-\lambda T}
$$

2) LOLP and EUE: LOLP are indices expressing generating system probability that cannot meet demand load. LOLP is calculated every hour by using ORR from units that are activated (committed) at that hour. EUE is described as a calculation of energy that is not able to be served by a generator. Unlike LOLP, EUE is calculated at the end of scheduling because it is a sum of each estimate of underserved load.

$$
\begin{aligned}
& L O L P_{t}=\sum_{j=1}^{n} P R_{j} L O S S_{j} \\
& E U E_{t}=\sum_{j=1}^{n} P R_{j} \operatorname{LOSS}_{j}\left(L O A D_{t}-C R_{j}\right)
\end{aligned}
$$

with

$$
\begin{aligned}
& \operatorname{LOSS}_{j}\left\{\begin{array}{l}
1, \quad \text { for } C R_{j}<\text { Load }_{t} \\
0, \quad \text { fot } C R_{j} \geq \operatorname{Load}_{t}
\end{array}\right. \\
& P R_{j} \quad=\text { COPT probability in } j \text { outage; }
\end{aligned}
$$

$$
\begin{array}{ll}
C R_{j} & =\text { in service capacity of COPT in } j \text { outage; } \\
\operatorname{Lad}_{t} & =\text { load demand at } t \text { hour; and } \\
n & =\text { total number of COPT table rows. }
\end{array}
$$

3) Load uncertainty: One of the objectives in combining load predicting uncertainty forecasting is to take into account inherent probabilities, namely the system load differs from the load predicting value. Load uncertainty is represented as a form of normal distribution with seven levels.

$$
P_{i}=P_{0}+(i \times S D)
$$

with

$P_{i}=$ estimation of the possible load;

$P_{0}=$ load demand occurring on that time;

$i \quad$ class interval; and

$S D=$ class' standard deviation of load prediction.

\section{NODAL ANT COLONY OPTIMIZATION}

ACO requires large computing space in its initialization process. A large number of all possible states $\left(2^{N}\right)$ is impossible to use it on a large scale. NACO is an ACO development created by changing initialization process in ACO by utilizing transition system existing in ACO [5]. This method breaks down states in ACO initialization process into several small parts called nodes. The nodes utilization in NACO is effective to reduce states possibility occurring in ACO, thus it can save memory capacity. Furthermore, nodes utilization also reduces a chance of state that has a total cost of each hour above the average.

NACO utilization also needed to be modified because there were differences in objective functions in its utilization. One modification that had been made was altering visibility values by using best cost per produced unit stated in [6]. These NACO simulation results were then used as final results in the study. Model of NACO Issues

UC initialization on either ACO or NACO had a very important role in its process. Importance of this initialization was because in those stages UC limitations were converted into states which were then utilized in transition process. The more states formed in this initialization could also increase optimal value of the utilized ACO method. Resolution of UC issues by utilizing NACO is broadly illustrated by a flowchart in Fig. 1.

\section{NACO Formulation}

NACO steps were generally divided into three, namely initialization, transition, and updating pheromone. These three steps repeated continuously until a desired condition was achieved.

1) NACO Initialization: NACO had a difference in its initialization state compared to regular ACO. In NACO initialization, generating units were broken down into small groups in their state-finding process. The separation was intended to simplify the combination of the previous state as much as $2^{N}$ per hour on ACO method to be $2^{N_{\text {nodal }}} x \frac{N}{N_{\text {nodal }}}$ with $N_{\text {nodal }}$ was number of generator in each part formed from generating unit. Using this method, the increase states number 
caused by combination due to number of generating units could be minimized, so as to cut down required memory capacity and computational time due to the search for states in ACO model.

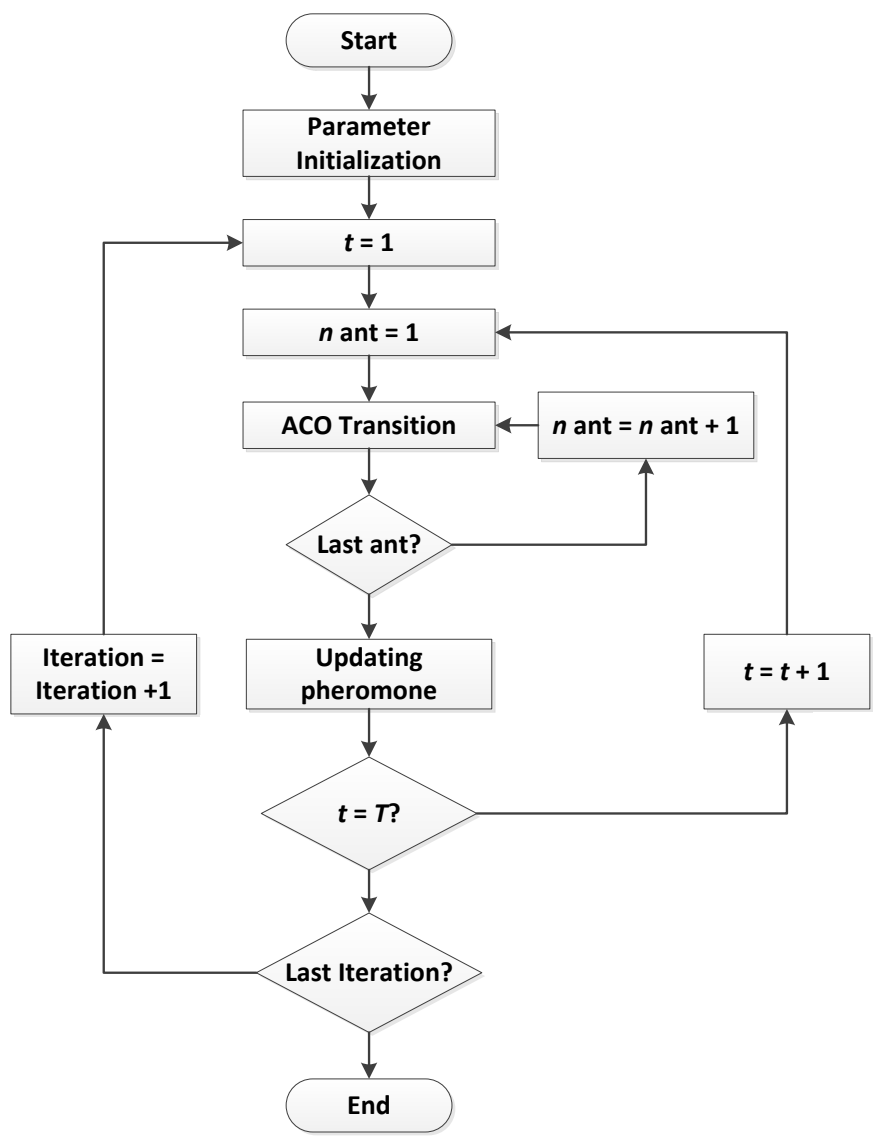

Fig. 1 NACO flowchart for UC issues.

In this process, NACO visibility formation made for ACO described how one state could be reached from a state where the ants were located [10]. In ACO, magnitude of visibility values was an inverse of possible transitional cost from this hour to the next hour.

$$
\eta_{(r, s)}=\frac{1}{F C_{(S)}+S U_{(r, s)}+\text { penalty }_{(r, s)}^{1}}
$$

Penalty given prior to transition would also affect a state's visibility level. One of UC penalties given at the beginning before transition was tmin-up/down penalty, in this study it was called as penalty ${ }^{1}$. It aimed to produce a collection of states meeting UC limitations, particularly tmin-up/down limitations.

2) NACO Transition: NACO transition in UC resolution was described as ants' displacement from an initial node to the next node. NACO transition was carried out randomly using roulette wheel method by considering probability of each state obtained from (12).

$$
P_{k}(r, s)=\frac{[\tau(s)][\eta(r, s)]^{\beta}}{\sum_{u \in s}[\tau(u)][\eta(r, u)]^{\beta}}, s \in S
$$

with

$$
\begin{array}{ll}
P_{k}(r, s)= & \text { transitional probability from } r \text { state to } s \text { state; } \\
\tau(s) & =\text { pheromone intensity on } s \text { state; } \\
\eta(r, s)= & \text { visibility from } r \text { state to } s \text { state; } \\
\beta & =\text { a parameter indicating the importance of } \\
& \text { pheromone against distance; } \\
S & =\text { a collection of states that allows a visit from } \\
& \text { previous state. }
\end{array}
$$

3) Pheromone Updating: Pheromone updating was a process to modify pheromone intensity existing inside pheromone matrix. Pheromone updating process in ACO was described as a process of adding or reducing pheromone intensity from each state so that it would affect visibility of that state in the next iteration.

$$
\tau(s)^{\prime}=(1-\varepsilon) \tau(s)+\Delta \tau(s)^{k}
$$

with

$\Delta \tau(s)^{k}=\left\{\begin{array}{cl}\frac{Q}{T C_{s}}, & \text { for a state passed by ants } k \\ 0, & \text { for other state values }\end{array}\right.$

$\tau(s) \quad=$ pheromone intensity on $s$ state;

$s \quad=$ selected states in transition process;

$\varepsilon \quad=$ evaporation factor, valued 0 to 1 ;

$Q \quad=$ constant; and

TC = transitional cost.

D. Best Cost per Produced utilization as a substitute for Fuel cost for NACO visibility

NACO had issues in utilizing fuel cost as visibility during transition process on each node. This issue occurred because the number of fuel cost could only be determined after economic dispatch was carried out, whereas in ACO dispatch could only be carried out if a state value had been formed. The impact on NACO was that during transition process between nodes, fuel cost visibility would be empty, so modifications were required to be carried out to substitute the value.

Best cost per produced [6] utilization in NACO in this study is used to substitute fuel cost value used as visibility. By entering fuel cost coefficient from each generator into (14), value of the best cost per produced for each generator is obtained.

$$
\operatorname{minCP} U_{i}=2 \sqrt{a_{i} c_{i}}+b_{i}\left(\frac{\$}{M W}\right)
$$

with

$C P U_{i}=$ cost per produced unit of generating unit $i$;

$a_{i}, b_{i}, c_{i}=$ fuel cost coefficient from generating unit $i$.

Then, the best production cost can be estimated without having to know the dispatched power. This the best cost per produced value is then used to substitute fuel cost value in (11) which is used as visibility. The generating unit is then sorted according to priority list based on best cost per produced value starting from the cheapest to the most expensive.

The best cost per produced will represent fuel cost of each generation in an active state based on priority order obtained 
from (14). Whereas in an inactive state, fuel cost is still taken using best cost per produced in a reverse order, that is, generator with first priority using the best cost per produced in the last priority generator in calculating its visibility. This needs to be done to prevent a void visibility value when the generator is off.

\section{E. Elitist Ant System Addition}

Elitist ant system addition carried out in NACO is used to improve optimization accuracy resulting from best cost per produced utilization in previous subsection, because the use of best cost per produced is not as accurate as fuel cost in describing visibility of a state. This lack of accuracy can cause the optimization process not to be as high as the previous ACO. Formation of elitist ant utilization is formulated in (15).

$$
\tau(s)^{k}=(1-\varepsilon) \tau(s)+\Delta \tau(s)^{k}+\Delta \tau(s)_{\text {best }}^{k}
$$

with

$$
\begin{aligned}
& \Delta \tau(s)^{k}=\left\{\begin{array}{cc}
\frac{Q}{T C_{s}}, & \text { for state that will be passed by ants } k \\
0, & \text { for other state values }
\end{array}\right. \\
& \Delta \tau(s)_{\text {best }}^{k}=\left\{\begin{array}{cc}
\sigma \frac{Q}{T C_{s}}, & \text { If the state is passed by }
\end{array}\right. \\
& \sigma \quad \text { for other state values } \\
& 0 \quad=\text { constants from elitist ants. }
\end{aligned}
$$

\section{Simulation Results}

UC issues simulations was carried out using MATLAB 2012 software, while data processing was carried out using a software from Microsoft, namely Microsoft Office Excel 2010 and Word 2010. The computer utilized for simulations used the AMD Phenom 965BE@ 3.7GHz processor with 8192MB RAM.

System test standard utilized I1996 IEEE Reliability Test System with 26 generators [9]. The utilized NACO referring to [5] as a basic theory guideline in using NACO. However, because the references used and this study had different objective functions, there were several modifications made, namely the utilization of the best cost per produced for EAS visibility and addition. Magnitude of pheromone matrix initial value was determined using a certain value, which was 0.001 . Value of the number of iterations, ant populations and evaporation referred to one website regarding optimization, i.e. 300, 50, and 0.05 [11].

In addition to obtaining the lowest cost, NACO modification was also carried out so that reliability constraints such as EUE and LOLP could be included in optimization process. Modifications made generally only altered the initialization process because the use of the best cost per produced and pheromones updating were due to EAS from the state. Another process such as transition process was still the same as the ACO transition process in general. Comparison results of IEEE
RTS1996 system simulation test against previous research are shown in Table I [7], [8].

TABLE I

COMPARISON OF GENERATING COST OF EACH METHOD

\begin{tabular}{|c|c|c|c|c|c|c|}
\hline $\begin{array}{c}\text { EUE } \\
(\mathbf{\%})\end{array}$ & $\begin{array}{c}\mathbf{L O L P} \\
\mathbf{( \% )}\end{array}$ & $\begin{array}{c}\text { LT } \\
\mathbf{( h )}\end{array}$ & $\begin{array}{c}\text { SD } \\
\mathbf{( \% )}\end{array}$ & $\begin{array}{c}\text { NACO } \\
\mathbf{( \$ )}\end{array}$ & $\begin{array}{c}\text { GA } \\
\mathbf{( \$ )}\end{array}$ & $\begin{array}{c}\text { SA } \\
\mathbf{( \$ )}\end{array}$ \\
\hline 0.10 & - & 4 & 0 & 708,537 & - & 708,789 \\
\hline 0.05 & - & 4 & 0 & 712,205 & - & 712,243 \\
\hline 0.01 & - & 4 & 0 & 717,995 & - & 718,747 \\
\hline- & 1.50 & 4 & 0 & 710,370 & 712,344 & 710,696 \\
\hline- & 1.00 & 4 & 0 & 717,592 & 719,225 & 717,938 \\
\hline- & 0.50 & 4 & 0 & 721,936 & 722,989 & 722,401 \\
\hline 0.05 & 1.5 & 2 & 0 & 708,818 & 709,112 & 708791 \\
\hline 0.05 & 1.5 & 4 & 0 & 711,712 & 712,680 & 712,067 \\
\hline 0.05 & 1.5 & 8 & 0 & 719,971 & 717,585 & 720,718 \\
\hline 0.01 & 1 & 2 & 0 & 715,962 & 715,782 & 716,083 \\
\hline 0.01 & 1 & 4 & 0 & 717,995 & 718,920 & 718,574 \\
\hline 0.01 & 1 & 8 & 0 & 722,085 & 722,532 & 723,300 \\
\hline 0.05 & 1.5 & 4 & 1 & 712,108 & 718,199 & 712,216 \\
\hline 0.05 & 1.5 & 4 & 3 & 713,085 & 713,802 & 713,855 \\
\hline 0.05 & 1.5 & 4 & 5 & 716,648 & 716,607 & 716,862 \\
\hline 0.01 & 1 & 4 & 1 & 718,351 & 719,102 & 718,843 \\
\hline 0.01 & 1 & 4 & 3 & 722,198 & 721,793 & 722,544 \\
\hline 0.01 & 1 & 4 & 5 & 728,583 & 722,373 & 730,675 \\
\hline
\end{tabular}

Table I compares the best total costs obtained from each method. The grey columns in the table show that the results shown by NACO are not the best cost. LT stands for lead time used to calculate system reliability based on ORR. SD is the standard deviation used in addition to load uncertainty.

In the modification of NACO visibility formed using the best cost per produced, premature convergence can occur due to pheromone intensity accumulation caused by formed visibility. Combination of EAS utilization tries to reduce emerging impact caused by the utilized visibility.

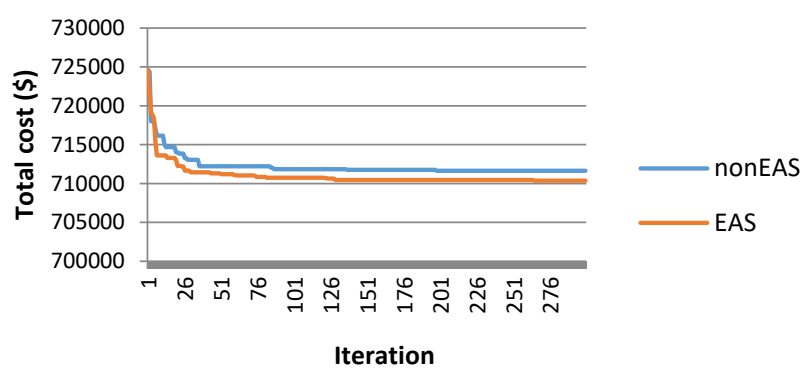

Fig. 2 Comparison of EAS and non-EAS simulation results.

Fig. 2 shows simulation results iteration shown from the utilization of EAS and non-EAS from NACO simulation proposed with the reliability of LOLP $1.5 \%$. EAS will force ants to make a transition to nodes that make up the best state in each hour, so that even if there is an increase in pheromone, the increase tends to occur at nodes that make up the best state in each hour. This method also reduces the impact of lack of occurring visibility accuracy, because pheromone intensity will slowly dominate transition process. 


\section{CONCLUSION}

In general, NACO simulation results with a comparison of GA and SA methods indicate that NACO is able to provide a relatively good result in its resolution. The use of best cost per produced as a substitute for fuel cost visibility and EAS addition is able to prove the results well. Simulations were carried out while maintaining system reliability shown in a form of LOLP and EUE indexes.

\section{REFERENCES}

[1] T. Logenthiran and D. Srinivasan, "Formulation of Unit Commitmen (UC) Problems and Analysis of Available Methodologies Used for Solving the Problems,” 2010 IEEE Int. Conf. Sustain. Energy Technol. ICSET 2010, 2010, pp. 1-6.

[2] M.Y. El-Sharkh, N.S. Sisworahardjo, A. Rahman, and M.S. Alam, “An Improved Ant Colony Search Algorithm for Unit Commitment Application,” 2006 IEEE PES Power Syst. Conf. Expo., 2006, pp. 17411746.

[3] T. Sum-im and W. Ongsakul, “Ant Colony Search Algorithm for Unit Commitment,” IEEE Int. Conf. Ind. Technol. 2003, 2003, pp. 72-77.

[4] K. Vaisakh and L.R. Srinivas, "Unit Commitment by Evolving Ant Colony Optimization,” World Congr. Nat. Biol. Inspired Comput., Vol. 3, pp. 67-77, 2010
[5] C.C. Columbus, K. Chandrasekaran, and S.P. Simon, "Nodal Ant Colony Optimization for Solving Profit Based Unit Commitment Problem for GENCOs,” Appl. Soft Comput. J., Vol. 12, No. 1, pp. 145-160, 2012.

[6] A.Y. Saber and A.M. Alshareef, "Scalable Unit Commitment by Memory-Bounded Ant Colony Optimization with A* Local Search,” Int. J. Electr. Power Energy Syst., Vol. 30, No. 6-7, pp. 403-414, 2008.

[7] D.R. Wijayanti, "Penjadwalan Pembangkit dengan Constraint Keandalan Menggunakan Algoritma Genetika Mempertimbangkan Ketidakpastian Beban”, Bachelor thesis, Universitas Gadjah Mada, Yogyakarta, Indonesia, 2014.

[8] D.N. Simopoulos, S.D. Kavatza, and C.D. Vournas, "Reliability Constrained Unit Commitment Using Simulated Annealing," IEEE Trans. Power Syst., Vol. 21, No. 4, pp. 1699-1706, 2006.

[9] D.N. Simopoulos, S.D. Kavatza, and C.D. Vournas, "Unit Commitment by an Enhanced Simulated Annealing Algorithm,” IEEE Trans. Power Syst., Vol. 21, No. 1, pp. 68-76, 2006.

[10] J. Heinonen and F. Pettersson, "Hybrid Ant Colony Optimization and Visibility Studies Applied to a Job-Shop Scheduling Problem,” Appl. Math. Comput., Vol. 187, No. 2, pp. 989-998, 2007.

[11] (2015) "Ant Colony Optimization" [Online], http://www.yarpiz.com/53/ypea103-ant-colony-optimization, access date: 15-Feb-2017. 